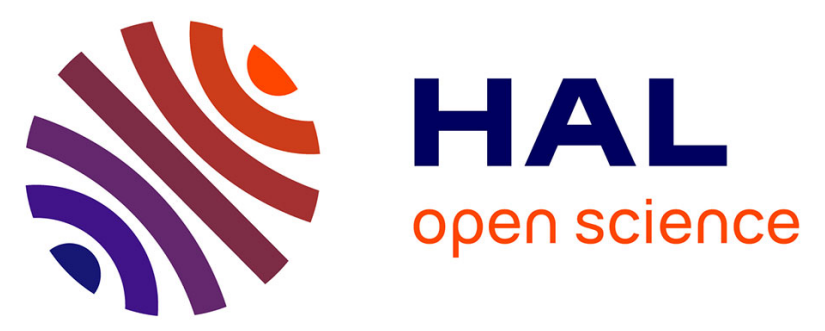

\title{
RÉSULTATS DE LA COLLABORATION SLAC-LBL A SPEAR
}

\author{
B. Jean-Marie, A. Boyarski, M. Breidenbach, F. Bulos, G. Feldman, G.
}

Fischer, D. Fryberger, G. Hanson, D. Hartill, R. Larsen, et al.

\section{- To cite this version:}

B. Jean-Marie, A. Boyarski, M. Breidenbach, F. Bulos, G. Feldman, et al.. RÉSULTATS DE LA COLLABORATION SLAC-LBL A SPEAR. Journal de Physique Colloques, 1976, 37 (C2), pp.C2-1C2-11. 10.1051/jphyscol:1976201 . jpa-00216473

\section{HAL Id: jpa-00216473 https://hal.science/jpa-00216473}

Submitted on 1 Jan 1976

HAL is a multi-disciplinary open access archive for the deposit and dissemination of scientific research documents, whether they are published or not. The documents may come from teaching and research institutions in France or abroad, or from public or private research centers.
L'archive ouverte pluridisciplinaire HAL, est destinée au dépôt et à la diffusion de documents scientifiques de niveau recherche, publiés ou non, émanant des établissements d'enseignement et de recherche français ou étrangers, des laboratoires publics ou privés. 


\title{
RÉSULTATS DE LA COLLABORATION SLAC-LBL A SPEAR
}

\author{
Présentés par B. JEAN-MARIE
}

A. M. Boyarski, M. Breidenbach, F. Bulos, G. J. Feldman, G. E. Fischer, D. Fryberger, G. Hanson, D. L. Hartill, B. Jean-Marie, R. R. Larsen, D. Lüke, V. Lüth, H. L. Lynch, D. Lyon, C. C. Morehouse, J. M. Paterson, M. L. Perl, T. P. Pun, P. Rapidis, B. Richter, R. F. Schwitters, W. Tanenbaum and F. Vannucci

Stanford Linear Accelerator Center Stanford University, Stanford, California 94305, U.S.A.

and

G. S. Abrams, D. D. Briggs, W. Chinowsky, C. E. Friedberg, G. Goldhaber, J. A. Kadyk, A. M. Litke, B. A. Lulu, F. M. Pierre, B. Sadoulet, G. H. Trilling, J. S. Whitaker, F. Winkelmann and J. E. Wiss

Lawrence Berkeley Laboratory and Department of Physics University of California, Berkeley, California 94720, U.S.A.

\begin{abstract}
Résumé. - Les résultats de l'étude de la section efficace d'annihilation $\mathrm{e}^{+} \mathrm{e}^{-}$, pour une énergie dans le centre de masse allant de 2,5 à 7,5 GeV, sont présentés. Aucune nouvelle structure comparable au $\psi(3095)$ et $\psi(3684)$ n'a été identifiée. La détermination des nombres quantiques des $\psi(3095)$ et $\psi(3684)$ est rapportée ainsi que les principaux modes de désintégration tels qu'ils sont connus à ce jour.
\end{abstract}

Abstract. - The results of the study of the $\mathrm{e}^{+} \mathrm{e}^{-}$cross section from 2.5 to $7.5 \mathrm{GeV}$ in the center of mass are presented. No new structure like $\psi(3095)$ and $\psi(3684)$ has been identified. The measurements of the $\psi(3095)$ and $\psi(3684)$ quantum numbers and their main decay modes are discussed.

1. Introduction. - Vouloir résumer en une heure les résultats obtenus à SPEAR par la collaboration SLAC-LBL est une gageure impossible à tenir. Aussi cet exposé s'attachera seulement à rappeler quelques résultats importants acquis et donnera des aperçus sur certains sujets plus neufs (donc plus intéressants). Pour ceux-ci la prudence est de rigueur car l'analyse n'en est pas achevée. Ils doivent être considérés comme des résultats préliminaires.

2. Description du détecteur. - Dans les anneaux de collision SPEAR [1], deux paquets, l'un d'électrons et l'autre de positrons, se croisent alternativement dans deux régions d'interaction. L'énergie des faisceaux peut varier de $1,3 \mathrm{GeV}$ à $4 \mathrm{GeV}$. La calibration absolue en énergie est $0,1 \%$ à $1,5 \mathrm{GeV}$. Les deux paquets ont une dispersion en énergie qui est supposée gaussienne avec $\sigma=1 \mathrm{MeV}$ à $1,5 \mathrm{GeV}$.

Le détecteur magnétique représenté dans la figure 1 est constitué d'un solénoïde de $3 \mathrm{~m}$ de long et de $3 \mathrm{~m}$ de diamètre développant un champ magnétique de $4 \mathrm{kG}$ qui est coaxial avec la direction des faisceaux. La trajectoire des particules chargées est reconstituée en employant les informations données par 4 ensembles de 4 chambres à fils magnétostrictives. Les fils sont inclinés à $\pm 2^{\circ}$ ou $\pm 4^{\circ}$ ce qui permet de mesurer les coordonnées azimutales $(\sigma=0,7 \mathrm{~mm})$ et longitudinales $(\sigma=14 \mathrm{~mm})$.

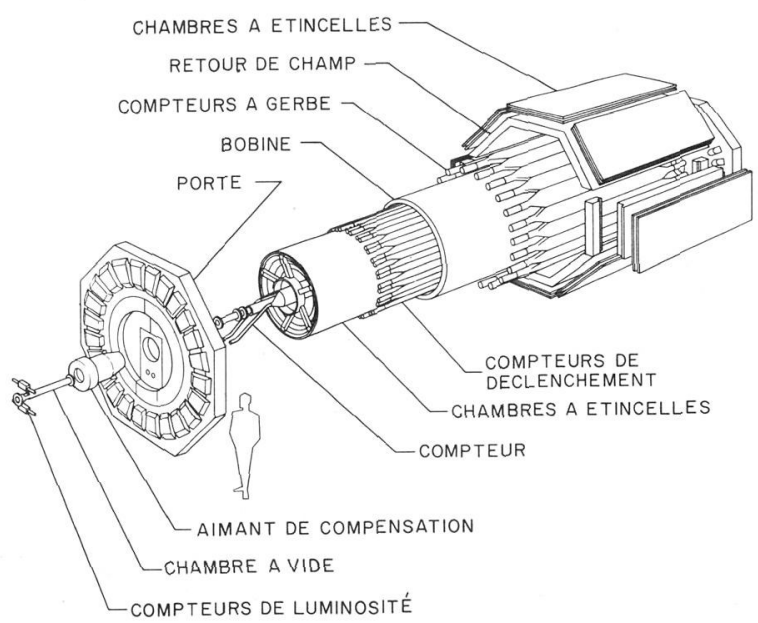

FIG. 1. - Vue télescopique du détecteur magnétique SLAC-LBL à SPEAR. 
Le déclenchement utilise les informations venant de 3 ensembles de compteurs.

D'abord une paire de compteurs à scintillation qui entourent la chambre à vide. Ensuite juste avant la bobine, est disposée une couronne de 48 compteurs à scintillation (utilisés aussi pour l'identification des particules par temps de vol). Finalement après la bobine 24 détecteurs sandwich permettent l'identification des électrons et la détection des gammas.

Les chambres sont déclenchées si au moins deux particules, après avoir ainsi traversé le premier compteur, donnent des signaux dans les compteurs de déclenchement et les compteurs à gerbe associés. Le retour de champ ( $20 \mathrm{~cm}$ d'acier) sert de filtre pour les particules hadroniques. Il est suivi de chambres planes magnétostrictives qui détectent les muons.

Le temps de vol permet de séparer $\pi, \mathbf{K}$ jusqu'à $600 \mathrm{MeV} / \mathrm{c}$ et $\mathrm{K}, \mathrm{p}$ jusqu'à $900 \mathrm{MeV} / \mathrm{c}$.

La normalisation des réactions est basée sur la mesure de la diffusion $\mathrm{e}^{+} \mathrm{e}^{-}$élastique détectée par deux ensembles de compteurs placés à petit angle le long du faisceau. Les compteurs sont calibrés en comparant avec la distribution à grand angle observée dans le détecteur dans des zones d'énergie où la contribution de graphes inélastiques est négligeable.

3. Annihilation $e^{+} e^{-}$entre 2,5 et $7,5 \mathrm{GeV}$. 3.1 LA SECTION EFFICACE TOTALE HADRONIQUE. L'étude de la section efficace totale d'annihilation $\mathrm{e}^{+} \mathrm{e}^{-}$constituait l'objectif principal de l'expérience.

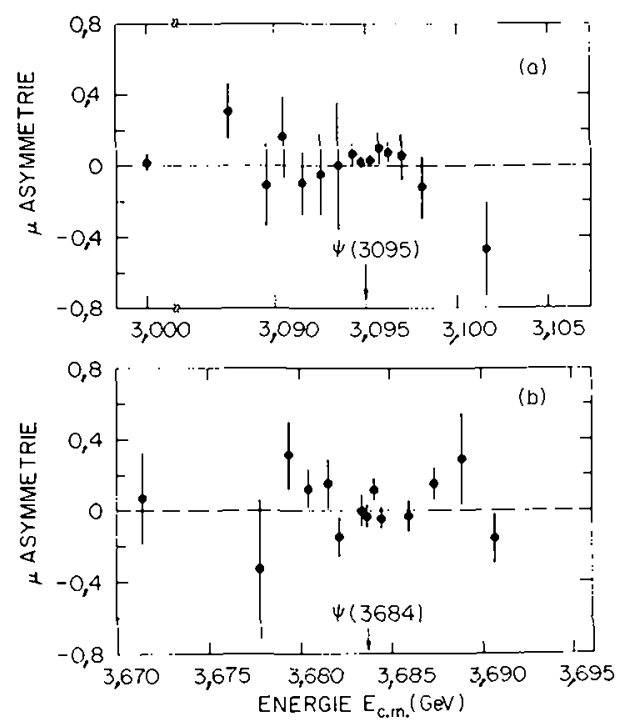

Fig. 2. - Section efficace totale d'annihilation $\mathrm{e}^{+} \mathrm{e}^{-}$entre 2,5 et $7,5 \mathrm{GeV}$.

Si l'on oublie, pour un temps, les résonances $\psi(3095)[2,3]$ et $\psi(3684)$ [4], on s'aperçoit que la section efficace totale entre 2,5 et $7,5 \mathrm{GeV}$ n'est pas dépourvue de surprises (Fig. 2). Certes elle décroît d'une façon générale de $30 \mathrm{nb}$ à $2,5 \mathrm{GeV}$ jusqu'à $10 \mathrm{nb}$ à $7,5 \mathrm{GeV}$. Cependant autour de $4,1 \mathrm{GeV}$, on observe une large structure qui fait penser à une résonance. En fait si l'on regarde la variation de :

$$
R=\frac{\sigma_{\mathrm{H}}^{\mathrm{TOTAL}}}{\sigma_{\mu \mu}}
$$

cette structure semble aussi très bien pouvoir s'interpréter comme un effet de seuil. Dans ce cas $R$ qui était constant entre 2,5 et $3,5 \mathrm{GeV}$, après avoir varié rapidement à $4,1 \mathrm{GeV}$, semble redevenir constant. Quelle est la signification de cette résonance ou de ce seuil ? Aucune réponse expérimentale ne peut encore être donnée car trop peu de données ont été recueillies à ce jour. La principale difficulté vient de ce que la section efficace au sommet n'est que de $30 \mathrm{nb}$. Si l'on considère que cette structure est une résonance, ses paramètres sont :

$$
\begin{aligned}
M & \simeq 4,1 \mathrm{GeV} \\
\sigma_{\mathrm{HAD}}^{\mathrm{PIC}} & \simeq 12 \mathrm{nb} \\
\Gamma & \simeq 240 \mathrm{MeV} .
\end{aligned}
$$

Il a été remarqué que la section efficace intégrée est :

$$
\int \sigma \mathrm{d} E=9900 \pm 1500 \mathrm{nb} \mathrm{MeV}
$$

Ceci se compare bien avec celles des $\psi(3095)$ et $\psi(3684)$ (voir tableau IV).

3.2 Multiplicité des particules Chargées. La variation de la multiplicité moyenne des particules chargées en fonction de l'énergie est montrée dans la figure 3. Elle est très bien représentée par une formule logarithmique :

$$
\left\langle n_{\mathrm{cb}}\right\rangle=1,93+1,5 \log E_{\mathrm{cm}} .
$$

Cette distribution est à peu près la même que celles observées dans la diffusion $\pi \mathrm{p}$ et dans l'annihilation $\mathrm{p} \overline{\mathrm{p}}$ [5]. On ne remarque pas de modification dramatique de la multiplicité autour de 4,1 GeV. Donc si un phénomène nouveau apparaît à cette énergie, il n'altère pas de façon sensible la multiplicité des particules chargées.

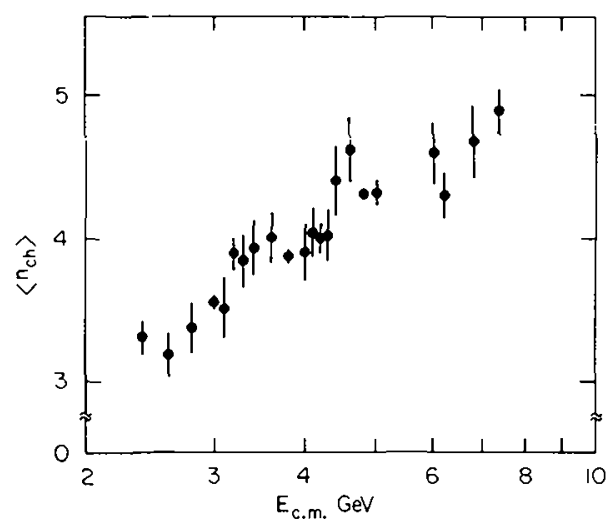

FIG. 3. - Multiplicité moyenne des particules chargées en fonction de l'énergie. Le fit donne $\left\langle n_{\mathrm{ch}}\right\rangle=1,93+1,5 \log E_{\mathrm{cm}}$. 
3.3 ObServation D'événements $\mu$ [6]. - Les données sont analysées de manière à isoler dans un événement une particule identifiée comme étant un électron (large signal dans les compteurs à gerbes et pas de trace dans les chambres extérieures) et l'autre étant un muon (pas de gerbe et une trace dans les chambres extérieures). Seuls sont pris en considération les couples de particules non coplanaires $\left(20^{\circ}\right)$ dont les moments sont supérieurs à $650 \mathrm{MeV} / \mathrm{c}$. Dans le tableau $I$, les événements sont classés suivant le nombre de photons associés, la charge totale et la multiplicité chargée. Les 24 événements e $\mu$ sans photons associés et de charge totale nulle attirent l'attention. On peut remarquer en effet qu'aucun événement ayant une charge totale \pm 2 n'a été trouvé. Si l'on s'intéresse à ces événements et si l'on essaie d'établir les différentes contributions provenant de mauvaises identifications entre les muons, les électrons et les hadrons, on obtient les nombres portés dans le tableau II.

\section{TABLEAU I}

Evénements satisfaisant les critères $\mathrm{e} \mu$. Ils sont classés suivant la charge totale, le nombre de photons observés et la multiplicité des particules chargées.

$\begin{array}{lrrrrrr}Q & 0 & 0 & 0 & \pm 2 & \pm 2 & \pm 2 \\ \text { Photons } & 0 & 1 & >1 & 0 & 1 & >1 \\ 2 \text { traces } & 24 & 8 & 8 & 0 & 0 & 3 \\ 3 \text { traces } & 5 & 7 & 21 & 3 & 2 & 2 \\ 4 \text { traces } & 5 & 4 & 8 & 3 & 5 & 7 \\ 5 \text { traces } & 3 & 2 & 4 & 4 & 5 & 3 \\ >5 \text { traces } & 2 & 3 & 4 & 1 & 2 & 1 \\ \text { Total } & 39 & 24 & 45 & 11 & 14 & 16\end{array}$

\section{TABlEAU II}

Evénements à deux traces. Les événements corrigés pour les mauvaises identifications e, $\mu$ sont comparés aux corrections calculées pour les mauvaises identifications des particules hadroniques.

$\begin{array}{lcccc}\text { Type } & \begin{array}{c}\text { BDF } \\ \text { ee }\end{array} & \begin{array}{c}\text { BDF } \\ \mu \mu\end{array} & \begin{array}{c}\text { Données } \\ \text { corrigées } \\ \text { pour ee, } \mu \mu\end{array} & \begin{array}{c}\text { BDF } \\ \text { HH }\end{array} \\ - & - & - & - & - \\ \text { ee } & 0 & 0 & 40 & 1,5 \pm 0,3 \\ \text { e } \mu & 1,0 \pm 1 & <0,3 & 0,23 \pm 0,5 & 3,3 \pm 0,6 \\ \mu \mu & 0 & 0 & 16 & 1,8 \pm 0,3 \\ \mathrm{eH} & 4,7 \pm 0,8 & 0 & 13,3 \pm 4,3 & 10,2 \pm 1,8 \\ \mu \mathrm{H} & 0 & 2,8 \pm 0,7 & 12,2 \pm 3,9 & 11,0 \pm 2,0 \\ \mathrm{HH} & 0,1 \pm 0,02 & 0 & 12,9 \pm 3,6 & \end{array}$

On s'aperçoit alors que les événements identifiés électron-hadron ou muon-hadron sont compatibles avec de mauvaises identifications. Tandis que les événements ee, $\mathrm{e} \mu, \mu \mu$ apparaissent à un niveau bien supérieur aux contaminations calculées. Il semble donc bien que l'on ait un signal réel. La figure 4 montre la distribution des impulsions des deux particules.

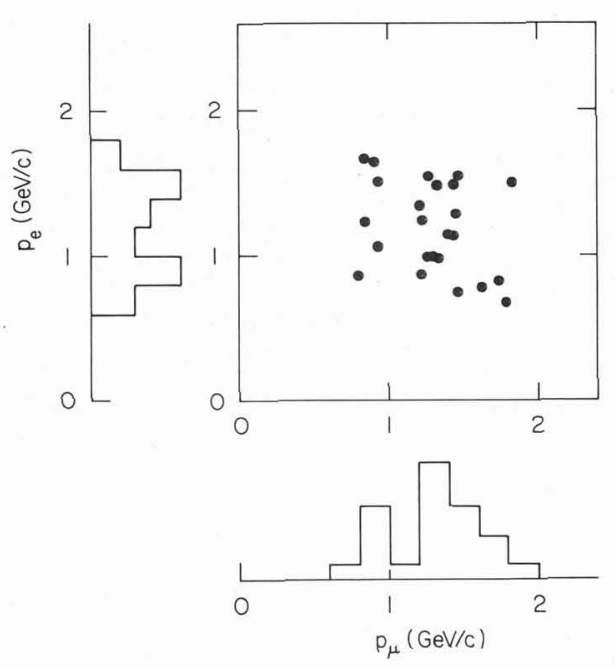

FIG. 4. - Distribution des moments des événements $\mathrm{e} \mu$.

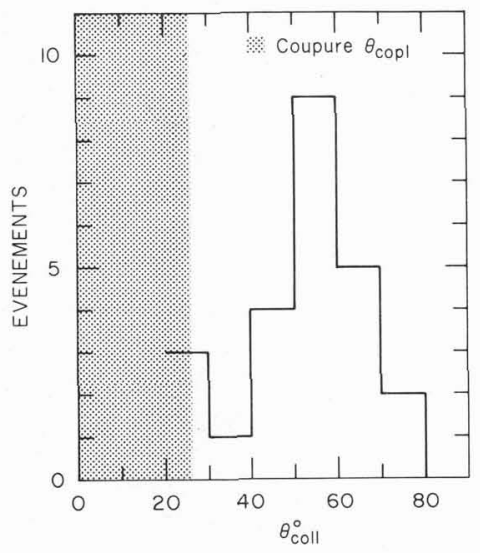

FIG. 5. - Distribution de l'angle entre les particules identifiées e, $\mu$. La coupure sur l'angle de coplanarité se réfléchit dans la distribution de colinéarité.

On remarque que les événements ne s'accumulent pas près des coupures. La figure 5 donne la distribution de colinéarité des particules. De nouveau les événements ne sont pas regroupés près des coupures. On peut donc penser que ces événements sont produits dans la réaction $\mathrm{e}^{+} \mathrm{e}^{-} \rightarrow \mathrm{X} \overline{\mathrm{X}}$ où $\mathrm{X} \rightarrow \mathrm{e}^{ \pm}+1$ ou plusieurs $v$ et $\overline{\mathrm{X}} \rightarrow \mu^{\mp}+1$ ou plusieurs $v$. Beaucoup d'hypothèses peuvent être avancées sur la nature de $\mathrm{X} \overrightarrow{\mathrm{X}}$. Il peut s'agir de la désintégration d'un lepton lourd, d'un méson lourd ou bien d'un boson intermédiaire. Trop peu d'informations sont recueillies pour trancher cette question. Cependant il est raisonnable de penser que l'on devrait observer :

$$
\mathrm{e}^{+} \mathrm{e}^{-} \rightarrow \mathrm{X} \overline{\mathrm{X}} \rightarrow \mathrm{e}^{+} \mathrm{e}^{-}+\text {neutres }
$$

ou

$$
\mathrm{e}^{+} \mathrm{e}^{-} \rightarrow \mathrm{X} \overline{\mathrm{X}} \rightarrow \mu^{+} \mu^{-}+\text {neutres . }
$$

Il semble qu'en effet de tels événements sont présents parmi les paires ee et $\mu \mu$ du tableau II.

Là encore davantage d'événements sont nécessaires afin de recueillir plus d'informations. 
3.4 ReCherChE DES PARTiCUl.eS Charmées [7]. Cette recherche a été faite, dans les données prises à $E_{\mathrm{cm}}=4,8 \mathrm{GeV} / \mathrm{c}$, en cherchant des variations dans le spectre de masse invariante de différentes associations de particules. Les figures 6 et 7 montrent les spectres pour $\pi^{+} \pi^{-}, \mathrm{K}^{+} \mathrm{K}^{-}, \mathrm{K}^{ \pm} \mathrm{K}^{\mp}, \pi^{+} \pi^{-} \pi^{+}, \mathrm{K}_{\mathrm{S}}^{0} \pi^{ \pm}$, $\mathbf{K}_{\mathbf{S}}^{0} \mathbf{K}^{ \pm}, \mathbf{K}^{ \pm} \pi^{+} \pi^{-}, \mathbf{K}_{\mathbf{S}}^{0} \pi^{+} \pi^{-}$. Les limites obtenues par mode varient de 0,1 à $0,5 \mathrm{nb}$. Elles montrent qu'il n'y a pas de désintégrations privilégiées dans aucun de ces modes. Elles sont cependant trop hautes pour tuer le modèle de charme.

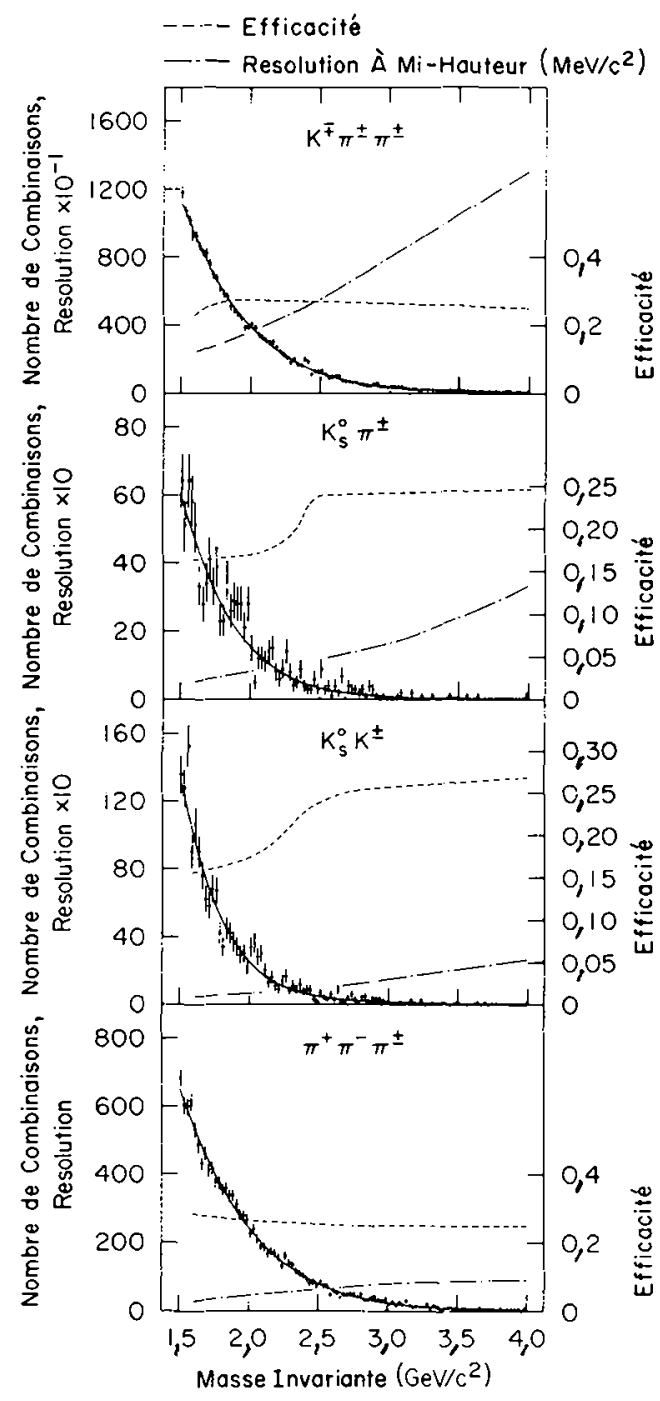

FIG. 6. - Distribution de masses invariantes pour différentes combinaisons de particules à l'énergie $E_{\mathrm{cm}}=4,8 \mathrm{GeV}$.

3.5 Observation de la polarisation des faisCEAUX. - On sait que les faisceaux tournant dans un anneau magnétique se polarisent [8] en alignant leur spin avec le champ magnétique. La polarisation à un moment donné est :

$$
P=P_{\operatorname{MAX}}\left(1-\mathrm{e}^{-t / \tau}\right)
$$

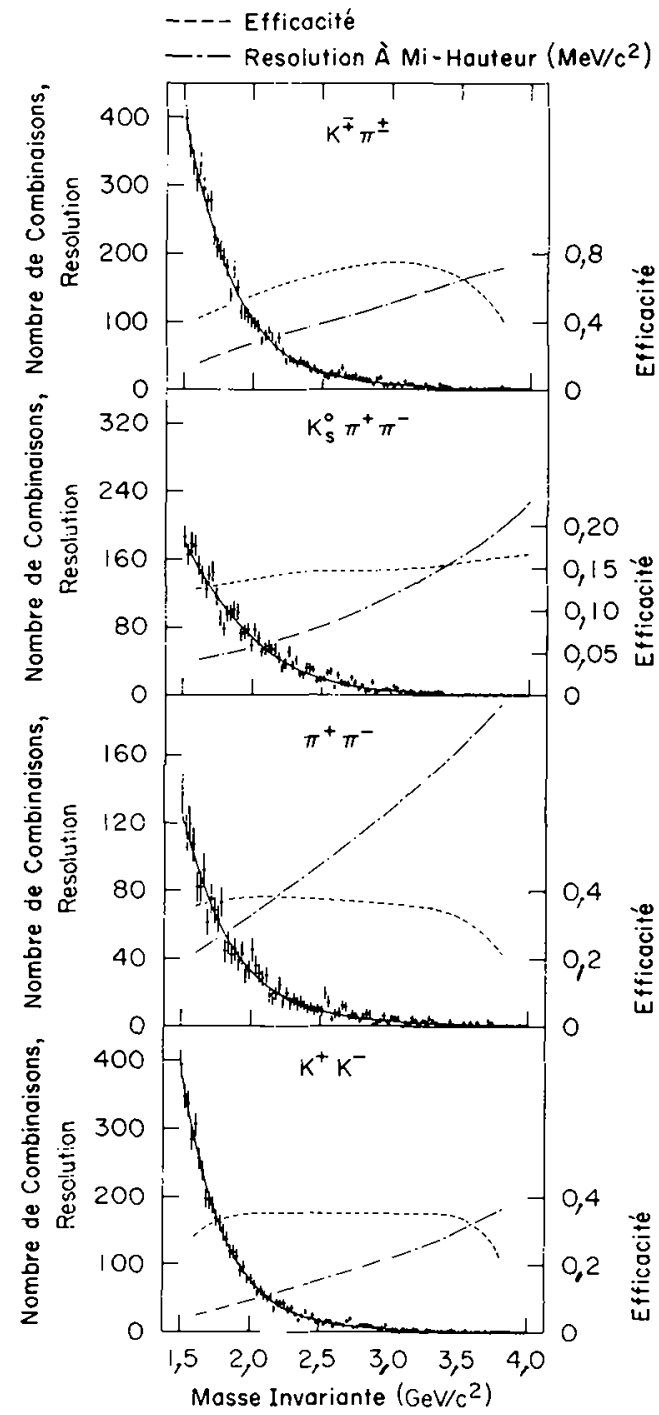

FIG. 7. - Distribution de masses invariantes pour différentes combinaisons de particules à l'énergie $E_{\mathrm{cm}}=4,8 \mathrm{GeV}$.

où $\tau$, qui est le temps de polarisation, dépend des caractéristiques magnétiques de l'anneau et varie comme $1 / E^{5}$. A l'énergie $E_{\mathrm{cm}}=7,4 \mathrm{GeV}$ le temps de polarisation est à peu près $14 \mathrm{~min}$. Cette polarisation introduit une asymétrie dans la distribution azimutale des particules. On peut le voir dans la figure $8 b$ donnant la distribution inclusive des particules en fonction de l'angle azimutal $\varphi$.

Par contre il est prévu que les faisceaux ne peuvent se polariser si le rapport $v$ entre la fréquence de précession du spin de l'électron et la fréquence de rotation des particules dans l'anneau est un nombre entier. A $E_{\mathrm{cm}}=6,181$ où $v=7,014$ on n'observe en effet aucune polarisation notable (Fig. $8 a$ ).

La section efficace pour l'échange à un photon avec des faisceaux polarisés s'écrit :

$$
\frac{\mathrm{d} \sigma}{\mathrm{d} \Omega}=1+\alpha \cos ^{2} \theta+\alpha\left|P_{+} P_{-}\right| \sin ^{2} \theta \cos 2 \varphi .
$$




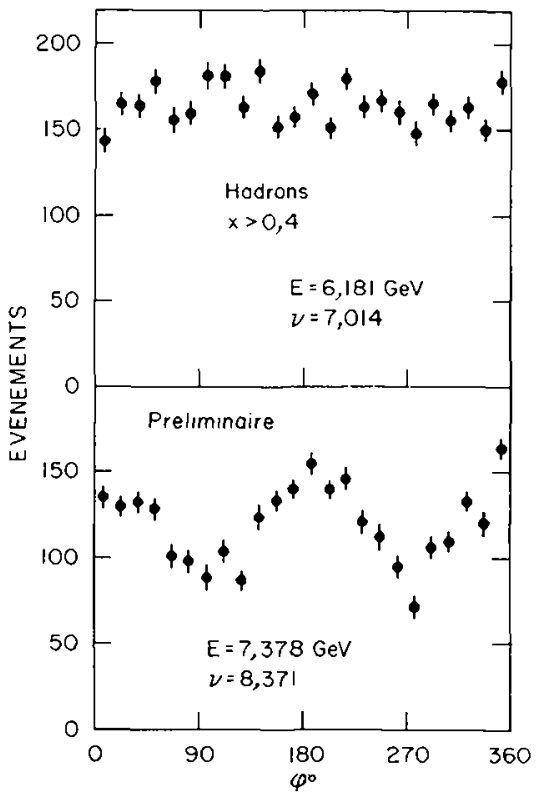

Fig. 8. $-a$ ) Distribution inclusive de l'angle azimutal des particules pour $E_{\mathrm{cm}}=6,181 \mathrm{GeV}$ et $X=P / P_{\mathrm{MAX}}$ supérieur à 0,4 . b) Même distribution pour $E_{\mathrm{cm}}=7,378 \mathrm{GeV}$. La polarisation est clairement visible.

On voit que la polarisation introduit une corrélation entre les angles $\theta$ et $\varphi$. Ceci peut être mis à profit pour étudier les distributions angulaires pour des valeurs de $\theta$ non couvertes par le détecteur. Dans ce sens la polarisation des faisceaux est un excellent outil expérimental.

4. Recherche de nouvelles résonances étroites. Après la découverte de la particule $\psi(3095)[2,3]$, une recherche systématique a été entreprise afin de trouver d'éventuelles résonances étroites du même type. La zone de $3,2 \mathrm{GeV}$ à $7,6 \mathrm{GeV}$ a été balayée par pas de $2 \mathrm{MeV}$ environ. Le temps passé à chaque point était calculé de manière à accumuler en moyenne 2 événements.

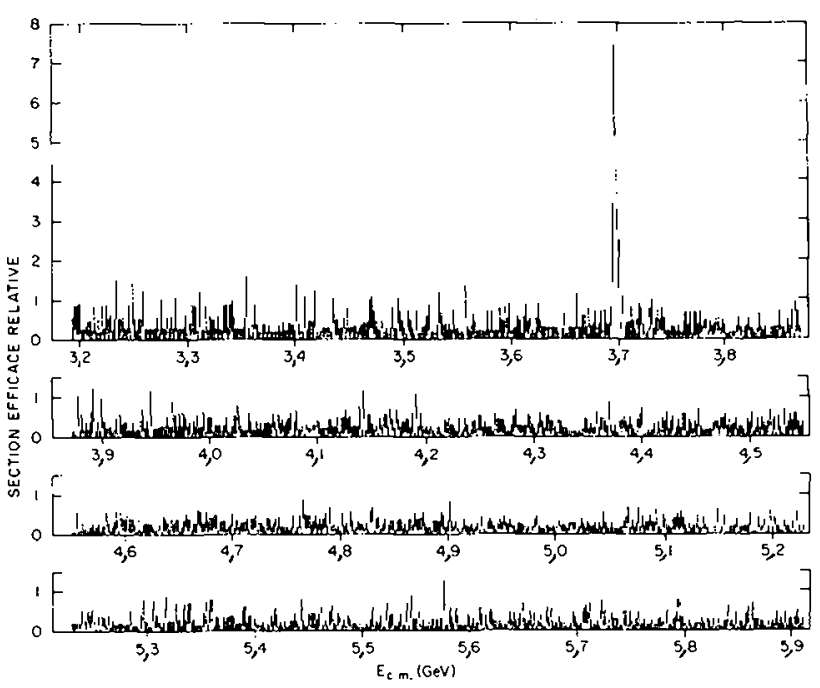

FiG. 9. - Section efficace relative, entre $3,2 \mathrm{GeV}$ et $5,9 \mathrm{GeV}$, mesurée en changeant l'énergie par pas de $2 \mathrm{MeV}$ environ.
L'analyse, faite en temps réel, permettait de déceler instantanément toute variation importante de la section efficace. Les résultats entre $3,2 \mathrm{GeV}$ et $5,9 \mathrm{GeV}$ sont présentés dans la figure 9 [9]. La sensibilité de cette recherche dépend de la largeur possible de la résonance. Le tableau III donne les limites supérieures pour la production de résonances étroites (inférieures à la résolution en énergie de la machine). A part la résonance $\psi(3684)$ découverte par cette méthode [2], aucune autre structure n'a été trouvée.

\section{TABLEAU III}

Limites supérieures pour la production de résonances étroites entre 3,2 et $7,6 \mathrm{GcV}$ dans l'hypothèse où la largeur de la résonance est plus petite que la résolution en énergie des faisceaux.

Domaine de masse

(GeV)

$$
\text { Limite sur } \int_{\text {HAD }} \mathrm{d} E_{\mathrm{cm}}
$$

\begin{tabular}{|c|c|c|}
\hline & - & - \\
\hline 3,20 & 3,50 & 97 \\
\hline 3,50 & 3,69 & $7 \varepsilon$ \\
\hline 3,72 & 4,00 & 8 \\
\hline 4,00 & 4,40 & 62 \\
\hline 4,40 & 4,90 & 58 \\
\hline 4,90 & 5,40 & 78 \\
\hline 5,40 & 5,90 & 8 \\
\hline 5,90 & 7,60 & \\
\hline
\end{tabular}

5. Propriétés des résonances $\psi(3$ 095) et $\psi(3$ 684) [ 10 , 11]. - Il est certain qu'en novembre 1974, la physique des annihilations $\mathrm{e}^{+} \mathrm{e}^{-}$est devenue très excitante. Il n'est pas difficile d'imaginer l'enthousiasme des expérimentateurs voyant en l'espace d'un $\mathrm{MeV}$ leur section efficace (et donc leur taux de comptage) multipliée par 100 !!

Ceci est bien en fait le mystère et la beauté de ces deux nouvelles résonances, que d'avoir une section efficace très grande et une largeur très étroite.

5.1 Mesure Des Sections efFicaces et DES LARGEURS. - La section efficace de production résonnante d'un état $f$ par annihilation $\mathrm{e}^{+} \mathrm{e}^{-}$a la forme

$$
\sigma_{\mathrm{f}}(E)=\frac{(2 J+1) \pi}{E^{2}} \frac{\Gamma_{\mathrm{e}} \Gamma_{\mathrm{f}}}{(M-E)^{2}-\Gamma^{2} / 4}
$$

où $J$ est le spin de la résonance,

$\Gamma_{\mathrm{e}}$ la largeur partielle en électrons,

$\Gamma_{\mathrm{f}}$ la largeur de l'état final considéré,

$\Gamma$ la largeur totale.

En fait compte tenu que les résonances sont plus étroites que la résolution en énergie des faisceaux, la seule quantité qui peut être mesurée expérimentalement est l'intégrale :

$$
\sigma_{\mathrm{f}}(E) \mathrm{d} E=\frac{2 \pi^{2}(2 J+1)}{M^{2}} \frac{\Gamma_{\mathrm{e}} \Gamma_{\mathrm{f}}}{\Gamma} .
$$


La mesure des sections efficaces partielles intégrées en hadron et lepton est alors suffisante pour déterminer tous les paramètres des résonances. On doit cependant supposer que $J=1$ (cela sera démontré par la suite) et que la largeur totale est :

$$
\Gamma=\Gamma_{\mathrm{H}}+\Gamma_{\mathrm{e}}+\Gamma_{\mu}=2 \Gamma_{\mu}+\Gamma_{\mathrm{H}}^{\mathrm{CHARGE}}
$$

Ceci implique que l'universalité $\mu \mathrm{e}$ est respectée, et qu'il n'y a pas de désintégrations des résonances en mode totalement neutre.

Les paramètres trouvés dans ces conditions pour les deux résonances sont présentés dans le tableau IV. L'universalité $\mu \mathrm{e}$ est testée et se révèle être vérifiée dans la limite des erreurs expérimentales.

\section{Tableau IV}

Paramètres des particules $\psi(3095)$ et $\psi(3684)$

$\begin{array}{ccc} & \psi_{3095} & \psi_{3684} \\ \text { Masse } & 3,095 \pm 0,004 \mathrm{GeV} & 3,684 \pm 0,005 \mathrm{GeV} \\ 1^{\mathrm{PC}} & 1-- & 1- \\ \int \sigma(E) \mathrm{d} E & 10400 \pm 1500 \mathrm{nb} \mathrm{MeV} & 3700 \pm 600 \mathrm{nb} \mathrm{MeV} \\ \int_{\mathrm{e}}=\Gamma_{\mu} & 4,8 \pm 0,6 \mathrm{keV} & 2,2 \pm 0,3 \mathrm{keV} \\ \Gamma_{\mathrm{H}} & 59 \pm 14 \mathrm{keV} & 220 \pm 56 \mathrm{keV} \\ \Gamma & 69 \pm 15 \mathrm{keV} & 225 \pm 56 \mathrm{keV} \\ \Gamma_{\mathrm{c}} / \Gamma & 0,069 \pm 0,009 & 0,0097 \pm 0,0016 \\ \Gamma_{\mathrm{H}} / \Gamma & 0,86 \pm 0,02 & 0,981 \pm 0,003 \\ \Gamma_{\mu} / \Gamma_{\mathrm{e}} & 1,00 \pm 0,05 & 0,89 \pm 0,16\end{array}$

Les erreurs tiennent compte de la statistique, de l'erreur sur l'efficacité calculée par Monte Carlo $(15 \%)$, de la normalisation $(3 \%)$ et de l'erreur dans la reproductibilité de l'énergie $(100 \mathrm{keV})$.

On remarque l'étroitesse des résonances. Le $\psi(3684)$ apparait environ 4 fois plus large. Cependant son rapport de branchement leptonique est 7 fois plus faible. Ceci est particulièrement remarquable en comparant les figures 10 et 11 . Les fits sont obtenus en tenant compte des corrections radiatives, des phénomènes d'interférences, et de la résolution en énergie des faisceaux. Ce sont les corrections radiatives qui sont responsables de l'allure dissymétrique des courbes.

5.2 DÉTERMINATION DES NOMBRES QUANTIQUES DE SPIN ET PARITÉ. - 5.2.1 Symétrie avant-arrière. Si les $\psi$ sont des états propres de $\mathbf{P}$ et de $\mathbf{C}$, il ne doit pas y avoir d'asymétrie dans la distribution angulaire des leptons.

La seule asymétrie qui peut être observée provient des corrections radiatives. Elle a été estimée à $2 \%$. L'asymétrie est étudiée sur les paires de $\mu$. Les rapports $\frac{H^{-} V}{H^{+} V}$ présentés dans la figure 12 montrent qu'aucune violation significative de $\mathrm{P}$ et $\mathrm{C}$ n'apparaît.

5.2.2 Détermination du spin $J=1$. - Comme il semble raisonnable de penser que les $\psi$ ont les mêmes

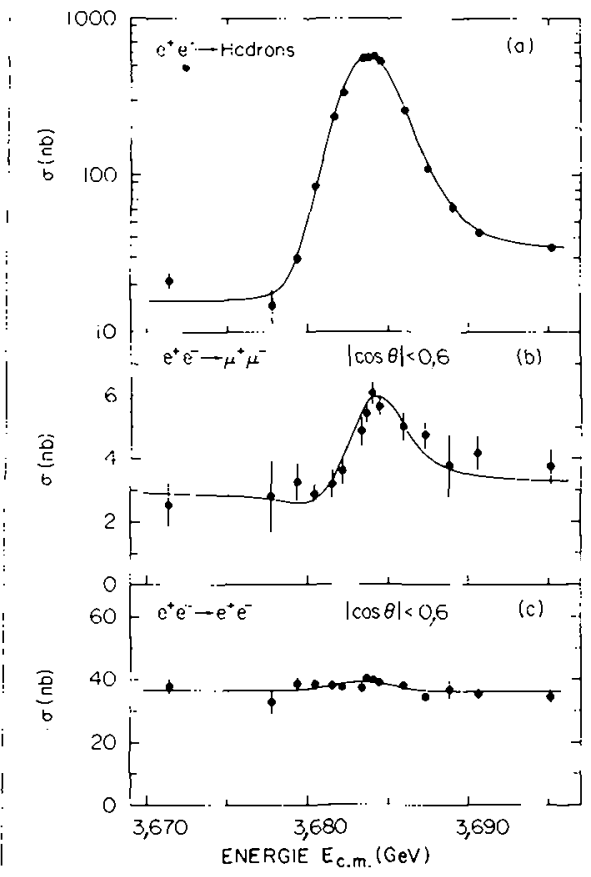

FIG. 10. - Sections efficaces $\sigma_{\mathrm{H}}, \sigma_{\mathrm{ee}}, \sigma_{\mu \mu}$ à la résonance $\psi(3095)$.

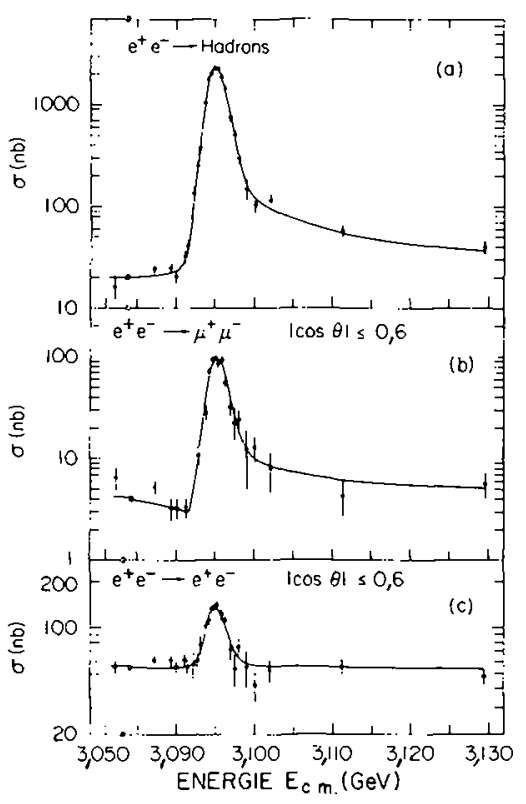

FIG. 11. - Sections efficaces $\sigma_{\mathrm{H}}, \sigma_{\mathrm{ee}}, \sigma_{\mu \mu}$ à la résonance $\psi(3684)$.

nombres quantiques que le photon, on doit s'attendre à observer une interférence entre les états finals produits par l'émission d'un photon virtuel et ceux produits à partir des $\psi$ par émission d'un deuxième gamma intermédiaire. Si l'on s'intéresse aux paires de $\mu$, la section efficace sera proportionnelle à :

$$
\sigma \simeq\left|-\frac{2 \alpha}{3}+\frac{\Gamma_{\mu}}{M-E_{\mathrm{cm}}-i \Gamma / 2}\right|^{2} .
$$




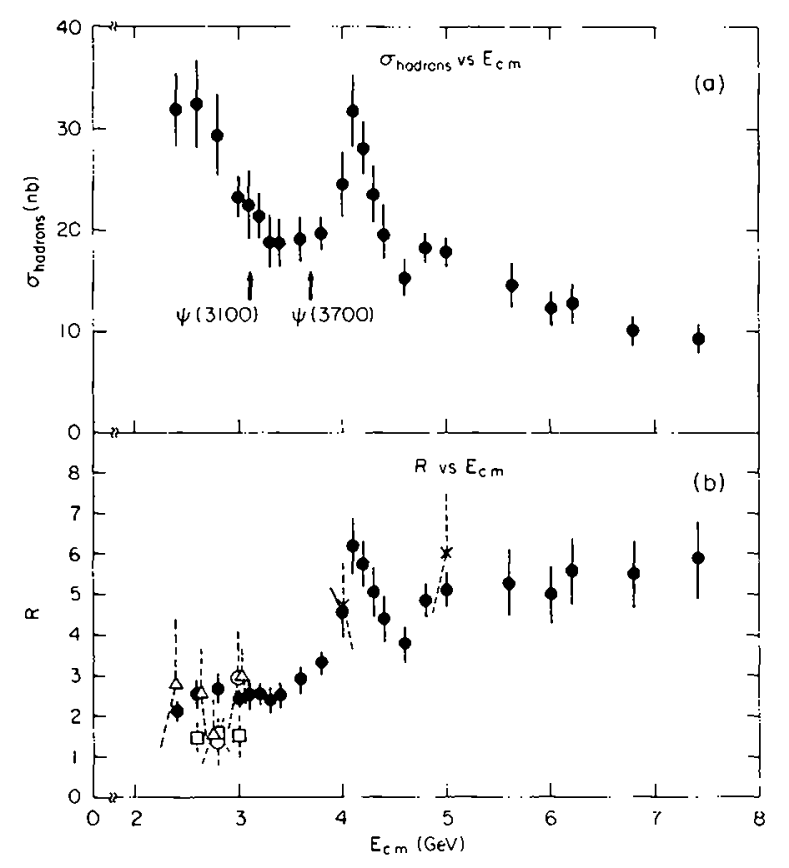

FIG. 12. - Asymétrie avant-arrière des paires de $\mu$. a) Dans la région de $\psi(3095)$. b) Dans la région de $\psi(3684)$.

On observera donc une interférence destructive avant la résonance et constructive après. En fait, afin de minimiser les erreurs systématiques, on préfère étudier le rapport :

$$
\frac{\sigma_{\mu \mu}}{\sigma_{\mathrm{e}^{+} \mathrm{e}^{-}}}
$$

L'effet d'interférence est en plus renforcé car il est constructif avant la résonance dans le cas des paires d'électrons. La figure 13 montre les résultats des fits obtenus sans interférence $(J=0)$ et avec interférence

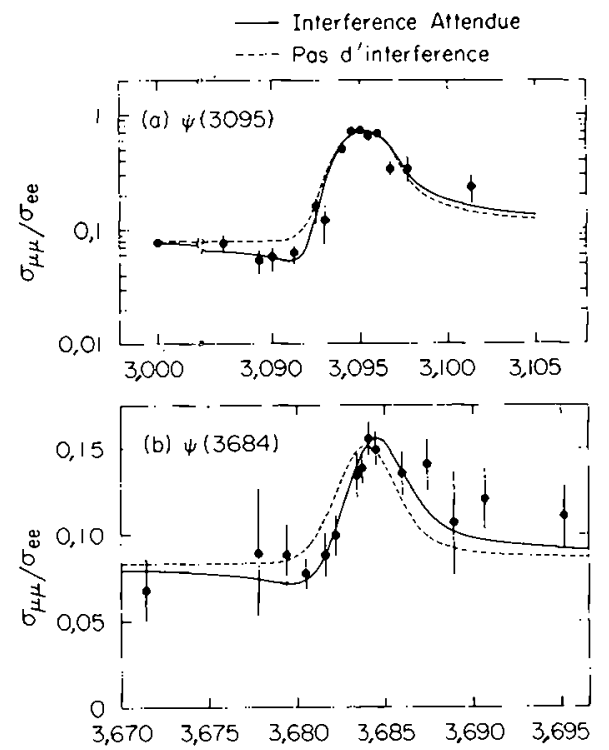

FIG. 13. - Distribution de $\sigma_{\mu \mu} / \sigma_{e e}$ en fonction de l'énergie; en trait plein, le fit en supposant une interférence totale $(J=1)$; en pointillé, le fit sans interférence $(J=0)$.
$(J=1)$. Cette seconde hypothèse est vérifiée par 2 et 5 écarts standard respectivement pour $\psi(3095)$ et $\psi(3684)$. Ce qui prouve que les $\psi$ ont les mêmes nombres quantiques que le photon. On peut remarquer en effet que si l'on avait $J=2$ ou $J=3$, l'interférence observée serait positive, $œ$ qui contredit les données. De plus les distributions angulaires $\mu \mu$ et ee (après soustraction de la part venant de la production Q.e.d.) sont en accord avec une forme $1+\cos ^{2} \theta$.

$5.3 G$ ParitÉ De $\psi(3$ 095) [12]. — Loin de la résonance

$$
R=\frac{\sigma_{\mathrm{H}}^{\mathrm{OFF}}}{\sigma_{\mu \mu}^{\mathrm{OFF}}}
$$

mesure le rapport entre la production des hadrons et celle des paires de $\mu$ par l'intermédiaire d'un photon virtuel. A la résonance, les paires de $\mu$ sont toujours produites par l'intermédiaire d'un photon virtuel (c'est-à-dire qu'il n'y a pas production directe $\psi \rightarrow \mu \mu$ ). Par contre les hadrons observés dans un état final donné f peuvent provenir soit :

- d'une désintégration directe du $\psi: \psi \rightarrow \mathrm{f}$, - d'une désintégration par l'intermédiaire d'un second photon virtuel $\psi \rightarrow \gamma \rightarrow \mathrm{f}$.

Dans ce dernier cas, le rapport :

sera égal à :

$$
R_{\mathrm{ON}}=\frac{\sigma_{\mathrm{f}}^{\mathrm{ON}}}{\sigma_{\mu \mu}^{\mathrm{ON}}}
$$

$$
R_{\mathrm{OFF}}=\frac{\sigma_{\mathrm{f}}^{\mathrm{OFF}}}{\sigma_{\mu \mu}^{\mathrm{OFF}}}
$$

car ce sont exactement les mêmes couplages qui interviennent. Par contre si l'état final observé provient d'une désintégration directe du $\psi$ on mesurera $R_{\text {ON }}>R_{\text {OFF. }}$. On a étudié la production de mode multipions en comparant les taux de production à l'énergie $3 \mathrm{GeV}$ et à l'énergie du $\psi$. On forme en fait le rapport :

$$
\alpha=\frac{R_{\mathrm{ON}}}{R_{\mathrm{OFF}}}=\frac{\sigma_{\mathrm{F}}^{\mathrm{ON}}}{\sigma_{\mathrm{F}}^{\mathrm{OFF}}} \times \frac{\sigma_{\mu \mu}^{\mathrm{OFF}}}{\sigma_{\mu \mu}^{\mathrm{ON}}}
$$

ce qui élimine la plupart des erreurs systématiques.

Les états étudiés sont :

$$
\begin{aligned}
& 2\left(\pi^{+} \pi^{-}\right), \quad 2\left(\pi^{+} \pi^{-}\right) 1 \pi^{0}, 3\left(\pi^{+} \pi^{-}\right), \\
& 3\left(\pi^{+} \pi^{-}\right) 1 \pi^{0} .
\end{aligned}
$$

La différence entre le comportement des états finals ayant un nombre impair ou pair de pions est frappante (Fig. 14). Ces derniers sont parfaitement compatibles avec une désintégration au deuxième ordre. Par contre les états finals avec un nombre impair de pions proviennent de désintégrations directes du $\psi$. Ceci suggère très fortement que la $G$ parité est un bon nombre quantique du $\psi$ et vaut $G=(-1)^{n}=-1$. 


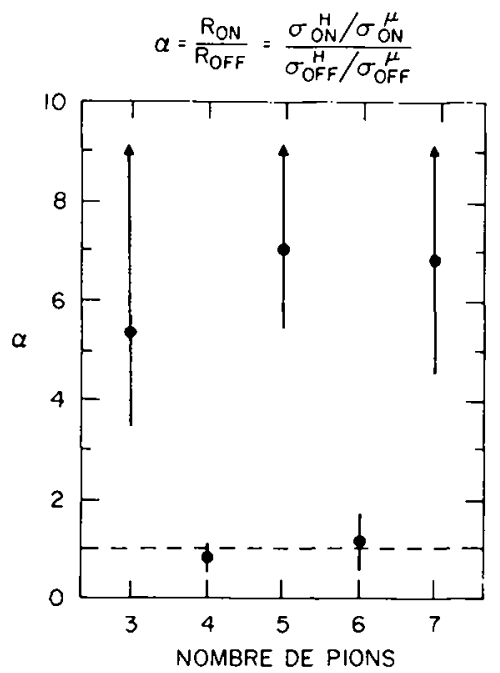

FIG. 14. - Comparaison des productions de multipions et des productions de paires de $\mu$, hors résonance et à la résonance $\psi(3095)$.

5.4 IsosPIN DE $\psi(3095)$. - Plusieurs arguments ont été développés pour déterminer la valeur de l'isospin de $\psi(3095)$.

Si l'on compare les taux de désintégration des 3 états de charge du mode $\psi \rightarrow \rho \pi$, on obtient suivant les valeurs de l'isospin du $\psi$ des prédictions très différentes. Si l'isospin est égal à zéro, on aura :

$$
\frac{\sigma_{\rho^{0} \pi^{0}}}{\sigma_{\rho^{+} \pi^{-}}+\sigma_{\rho^{-} \pi^{+}}}=0,5 \text {. }
$$

Ce même rapport vaudra 2 si l'isospin est égal à 2 . (1 est exclu à cause de la $G$ parité négative, car $G=C(-1))^{1}$.

Le mode $\psi \rightarrow \rho \pi$ a été effectivement observé parmi les événements $\pi^{+} \pi^{-} \pi^{0}$. La figure 15 montre dans le diagramme de Dalitz.des 3 pions, les bandes caractéristiques correspondant aux trois états de charge du $\rho$. On trouve expérimentalement :

$$
\frac{\sigma_{\rho^{0} \pi^{0}}}{\sigma_{\rho^{+} \pi^{-}}+\sigma_{\rho^{-} \pi^{+}}}=0,59 \pm 0,17 \text {. }
$$

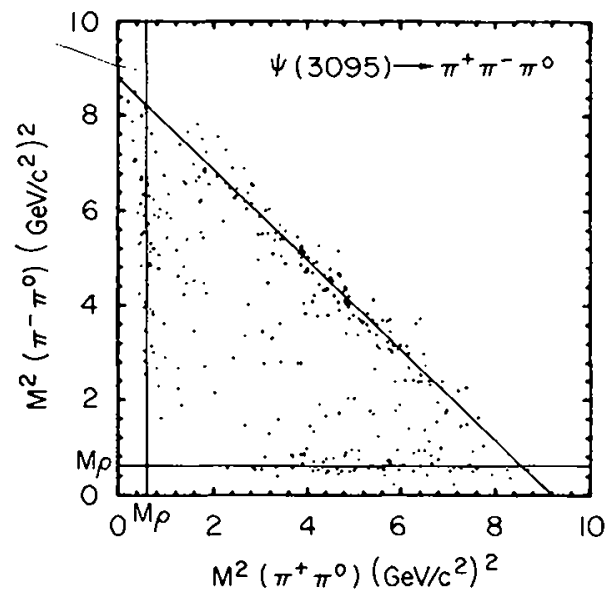

FiG. 15. - Diagramme de Dalitz des événements $\pi^{+} \pi^{-} \pi^{0}$ venant du $\psi(3095)$.
Ce qui détermine que l'isospin du $\psi(3095)$ est égal à zéro.

Un autre argument peut être développé en employant les désintégrations baryoniques du $\psi$ :

$$
\psi \rightarrow \mathrm{p} \overline{\mathrm{p}} \text { et } \psi \rightarrow \Lambda \bar{\Lambda} .
$$

Les premiers sont séparés facilement des paires $\mu^{+} \mu^{-}$par un fit contraint des deux traces (Fig. 16). Les seconds sont trouvés dans les événements $\pi \pi \mathrm{p} \overline{\mathrm{p}}$. La reconstruction des deux masses de $A$ permet une identification, sans bruit de fond, du signal (Fig. 17). Les états de spin isotopique de ces systèmes ne peuvent être que 0 ou 1 . A cause de la parité $G$ qui est négative, le seul état possible est donc : $I=0$.

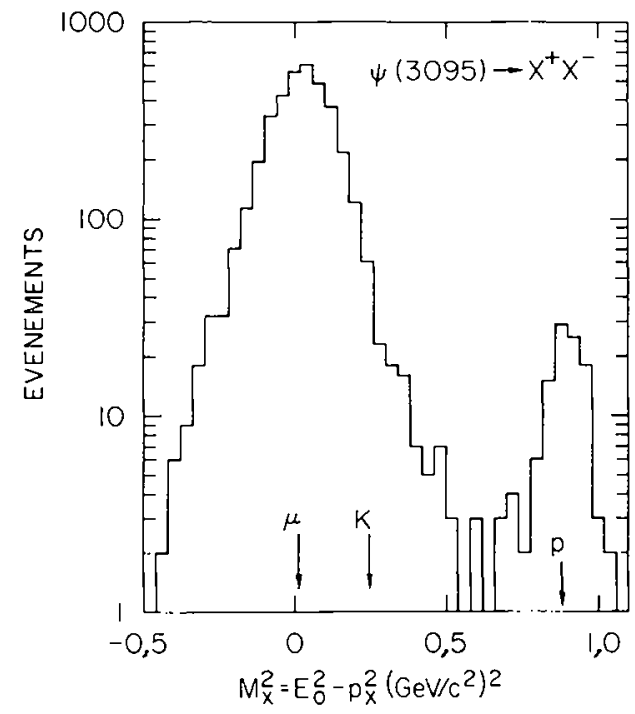

Fig. 16. - a) Masse invariante du système $\pi^{+} \pi^{-}$pour les événements détectés par 2 traces chargées de charge totale zéro. $b$ ) Pour ceux des événements tels que $M_{\pi^{*} \pi^{-}}=M_{\mathrm{K}_{\mathrm{g}}}$ distribution de la masse manquantc du système. Un seul événement est trouvé à la masse du $\mathrm{K}_{\mathrm{i}}^{0}$.

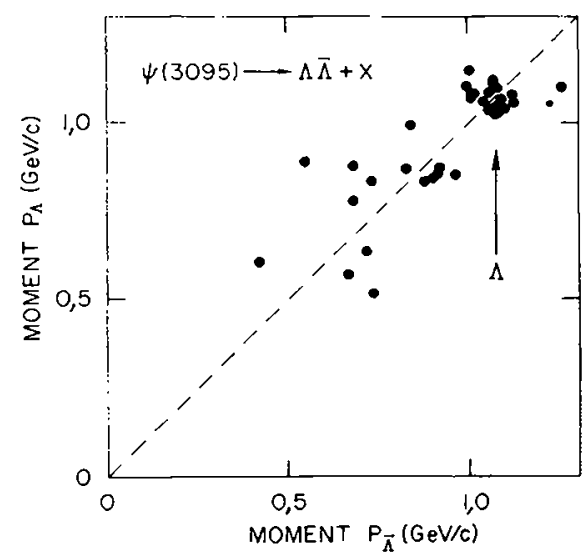

Fig. 17. - Distribution de $P_{A}$ par rapport à $P_{\bar{A}}$ pour les événements $\psi \rightarrow \pi^{+} \pi^{-} \mathrm{p} \overline{\mathrm{p}}$ 


\section{TABLEAU $\mathrm{V}$}

Modes de désintégration de $\psi(3095)$

\begin{tabular}{|c|c|c|c|}
\hline Mode & $\begin{array}{c}\text { Rapport } \\
\text { de branchement } \\
--\end{array}$ & $\begin{array}{c}\text { Evénements } \\
-\end{array}$ & $\begin{array}{c}\text { Commentaires } \\
-\end{array}$ \\
\hline $\begin{array}{l}\mathrm{e}^{+} \mathrm{e}^{-} \\
\mu^{+} \mu^{-}\end{array}$ & $\begin{array}{l}6,9 \pm 0,9 \\
6,9 \pm 0,9\end{array}$ & $\begin{array}{l}\sim 2000 \\
\sim 2000\end{array}$ & \\
\hline $\begin{array}{l}\eta \varphi \\
\eta \pi \pi\end{array}$ & & & $\begin{array}{c}\text { vue } \\
\text { limite supérieure }\end{array}$ \\
\hline $\begin{array}{l}\rho \pi \\
2 \pi^{+} 2 \pi^{-} \\
2 \pi^{+} 2 \pi^{--} \pi^{0} \\
3 \pi^{+} 3 \pi^{--} \\
3 \pi^{+} 3 \pi^{-} \pi^{0} \\
4 \pi^{+} 4 \pi^{-} \pi^{0}\end{array}$ & $\begin{array}{l}1,3 \pm 0,3 \\
0,4 \pm 0,1 \\
4,0 \pm 1,0 \\
0,4 \pm 0,2 \\
2,9 \pm 0,7 \\
0,9 \pm 0,3\end{array}$ & $\begin{array}{r}153 \pm 13 \\
76 \pm 9 \\
675 \pm 40 \\
32 \pm 7 \\
181 \pm 26 \\
13 \pm 4\end{array}$ & $\left\{\begin{array}{l}20 \% \omega \pi^{+} \pi^{-} \\
30 \% \rho \pi \pi \pi\end{array}\right.$ \\
\hline $\begin{array}{l}\pi^{+} \pi^{-} \mathbf{K}^{+} \mathbf{K}^{-} \\
\pi^{+} \pi^{-} \mathbf{K}^{+} \mathbf{K}^{-} \pi^{0} \\
2 \pi^{+} 2 \pi^{--} \mathbf{K}^{+} \mathbf{K}^{-}\end{array}$ & $\begin{array}{c}0,4 \pm 0,2 \\
-- \\
0,3 \pm 0,2\end{array}$ & $\begin{array}{c}83 \pm 18 \\
- \\
-\end{array}$ & $\begin{array}{c}\text { non } \mathrm{K}_{892}^{*} \mathrm{~K}_{1420}^{*} \\
\text { vue }\end{array}$ \\
\hline $\begin{array}{l}\pi^{+} \pi^{-} \mathrm{K}_{\mathrm{S}}^{0} \mathrm{~K}^{ \pm} \pi^{\mp} \pi^{0} \\
\mathrm{~K}_{\mathrm{S}} \mathrm{K}_{\mathrm{L}} \\
\mathrm{K}^{0} \mathrm{~K}^{*}(892)\end{array}$ & $\begin{array}{c}<\overline{0,02} \\
0,24 \pm 0,05\end{array}$ & $\begin{array}{c}- \\
\leqslant 1 \\
57 \pm 12\end{array}$ & $\begin{array}{c}\text { vue } \\
\mathrm{K}^{0} \frac{90}{\mathrm{~K}^{*}}+\overline{\mathrm{K}}^{0} \mathrm{~K}^{0 *}\end{array}$ \\
\hline $\mathrm{K}^{ \pm} \mathrm{K}^{\mp *}(892)$ & $0,31 \pm 0,07$ & $87 \pm 19$ & $\mathrm{~K}^{+} \mathrm{K}^{-*}+\mathrm{K}^{-} \mathrm{K}^{*+}$ \\
\hline $\mathrm{K}^{0} \mathrm{~K}^{0 *}(1420)$ & $<0,19$ & $\leqslant 3$ & $90 \%$ C.L. \\
\hline $\begin{array}{l}\mathrm{K}^{ \pm} \mathrm{K}^{\mp *}(1420) \\
\mathrm{K}^{* 0}(892) \overline{\mathrm{K}^{* 0}(892)}\end{array}$ & $\begin{array}{l}<0,19 \\
<0,06\end{array}$ & $\begin{array}{l}\leqslant 3 \\
\leqslant 3\end{array}$ & $\begin{array}{l}90 \% \text { C.L. } \\
90 \% \text { C.L. }\end{array}$ \\
\hline $\mathrm{K} * 0(1420) \bar{K}^{* 0}\left(\begin{array}{lll}1 & 420)\end{array}\right.$ & $<0,18$ & $\leqslant 3$ & $90 \%$ C.L. \\
\hline $\mathrm{K}^{* 0}(892) \mathrm{K}^{* 0}(1420)$ & $0,37 \pm 0,10$ & $30 \pm 7$ & \\
\hline $\begin{array}{l}\mathrm{p} \overline{\mathrm{p}} \\
\Lambda \bar{\Lambda} \\
\mathrm{p} \overline{\mathrm{p}} \pi^{0}\end{array}$ & $\begin{array}{l}0,21 \pm 0,04 \\
0,16 \pm 0,08\end{array}$ & $\begin{array}{r}105 \pm 11 \\
19 \pm 5\end{array}$ & \\
\hline $\begin{array}{l}\overline{\mathrm{n}} \mathrm{p} \pi^{-} \\
\overline{\mathrm{p}} \mathrm{n} \pi^{+}\end{array}$ & $0,37 \pm 0,19$ & $87 \pm 30$ & \\
\hline
\end{tabular}

5.5 MODES DE DÉSINTÉGRATIONS HADRONIQUES DE $\psi(3095)$. - Le $\psi(3095)$ se révèle être riche en désintégrations hadroniques. Quelques-unes sont rapportées dans le tableau $V$. Le fait que le mode est vu ne veut pas dire qu'il soit faible mais seulement qu'il n'a pas encore été étudié.

Les systèmes de multipions, dont on a déjà parlé, semblent avoir les mêmes comportements que leurs homologues des annihilations $\mathrm{p} \overline{\mathbf{p}}$. En particulier la production de $\rho, \omega$, f est observée.

Les modes comportant des $\mathrm{K}$ chargés ou neutres ont également été étudiés et la production de $\mathrm{K}_{900}^{*}$ et $\mathrm{K}_{1400}^{*}$ est abondante.

C'est en étudiant les désintégrations hadroniques que l'on peut essayer de vérifier que $\psi(3095)$ est un singlet de SU(3). En effet, dans ce cas $\psi(3095)$ ne peut pas se désintégrer en deux particules appartenant au même multiplet. Par exemple les désintégrations $\psi \rightarrow \mathrm{K}^{+} \mathrm{K}^{-}, \psi \rightarrow \pi^{+} \pi^{-}, \psi \rightarrow \mathrm{K}_{\mathrm{S}}^{0} \mathrm{~K}_{\mathrm{L}}^{0}$ sont interdites. Aucune d'elles n'a été observée. De l'étude du signal $\mathrm{K}_{\mathrm{S}}^{0}$ dans les événements détectés avec deux traces (Fig. 18),

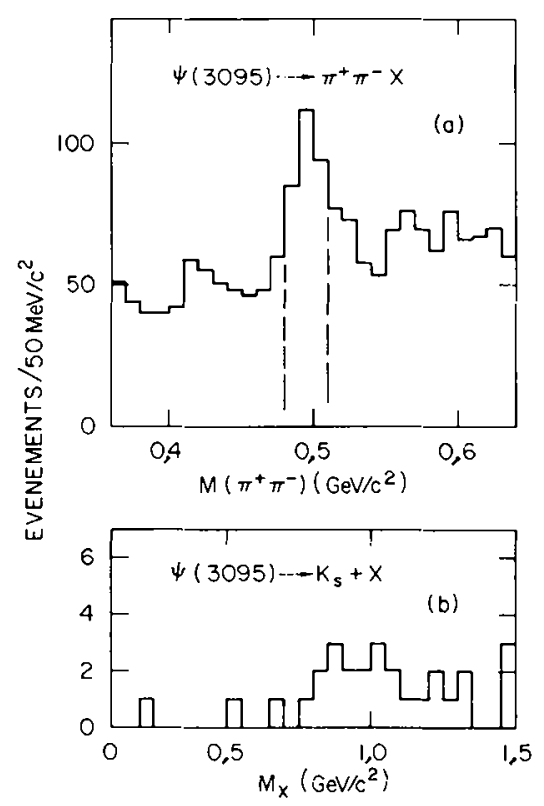

FIG. 18. - Masse carrée invariante pour les événements ayan deux traces colinéaires. 
on peut donner une limite supérieure du rapport de branchement :

$$
B\left(\psi \rightarrow \mathrm{K}_{\mathrm{S}}^{0} \mathrm{~K}_{\mathrm{L}}^{0}\right)<0,02 \% \quad 90 \% \text { C.L. }
$$

De même l'égalité entre les désintégrations $\psi \rightarrow \mathrm{p} \overline{\mathrm{p}}$ et $\psi \rightarrow \Lambda \bar{A}$ vérifie bien les prédictions de SU(3).

Par contre la prédiction :

$$
\sigma_{\rho \pi}=\frac{3}{4} \sigma_{\mathrm{KK} *}
$$

semble violemment fausse.

5.6 DÉSINTÉGRATIONS HADRONIQUES DE $\psi(3684)$. Le mode principal de désintégration du $\psi(3684)$ se fait par la cascade :

$$
\psi(3684) \rightarrow \pi \pi \psi(3095) \text {. }
$$

Le mode est aisément trouvé dans la réaction :

$$
\psi(3684) \rightarrow \pi^{+} \pi^{-}+\text {quelque chose . }
$$

La figure 19 montre la masse qui est opposée au système $\pi^{+} \pi^{-}$pour l'ensemble des événements $(a)$ et pour un sous-ensemble d'événements à quatre traces $(b)$, dont deux d'entre elles ont été identifiées électrons ou muons. On peut calculer le rapport d'embranchement [13] :

$$
A=\frac{\psi(3684) \rightarrow \pi^{+} \pi^{-} \psi(3095)}{\psi(3684) \rightarrow \text { total }}=0,32 \pm 0,04 .
$$

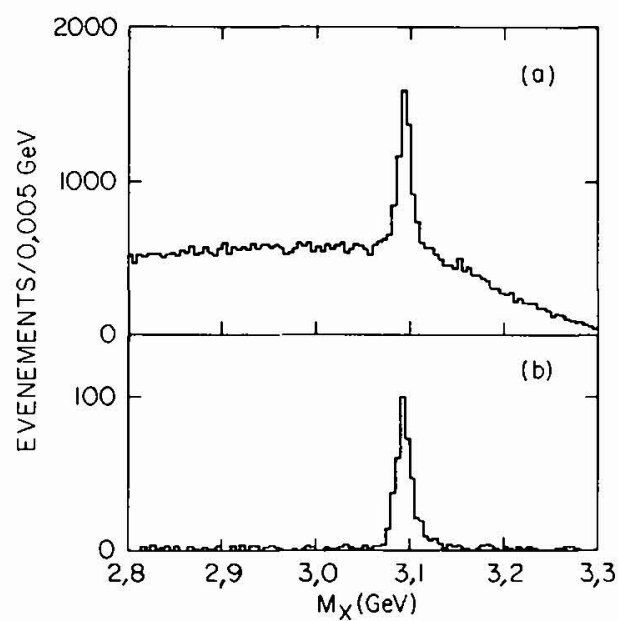

FIG. 19. - a) Masse manquante opposée au système $\pi^{+} \pi^{-}$pour l'ensemble des événements. b) Masse manquante opposée au système $\pi^{+} \pi^{-}$quand les 2 particules opposées aux pions sont identifiées électrons ou muons.

De même de l'étude de :

$$
\psi(3684) \rightarrow \psi(3095)+\text { quelque chose }
$$

où $\psi(3095)$ est détecté par sa désintégration en $\mu^{+} \mu^{-}$, on déduit :

$$
\begin{aligned}
B & =\frac{\psi(3684) \rightarrow \psi(3095)+\text { quelque chose }}{\psi(3684) \rightarrow \text { total }} \\
& =0,57 \pm 0,08 .
\end{aligned}
$$

Suivant que l'isospin est 0,1 ou 2 on s'attend à ce que le rapport $A / B$ vaille $1,52,1$ ou 3,1 . Là encore la valeur de l'isospin $I=0$ est clairement déterminée car expérimentalement $A / B=1,78 \pm 0,10$. Il est cependant important de remarquer que la différence entre les valeurs prédites et mesurées peut seulement s'expliquer par la présence d'autres transitions que

ou

$$
\begin{aligned}
& \psi(3684) \rightarrow \pi^{+} \pi^{-} \psi(3095) \\
& \psi(3684) \rightarrow \pi^{0} \pi^{0} \psi(3095) .
\end{aligned}
$$

On peut estimer qu'environ $10 \%$ des désintégrations du $\psi(3$ 684) peuvent se faire via $\psi(3684) \rightarrow \eta \psi(3095)$ ou $\psi(3684) \rightarrow$ neutre $\psi(3095)$. A ce jour de sérieuses évidences ont été trouvées pour le mode $\psi(3684) \rightarrow \eta \psi(3$ 095) $(4 \pm 2 \%)$. De même une active recherche est faite pour identifier des transitions du type $\psi(3684) \rightarrow \gamma \gamma \psi(3095)$.

De tels modes sont en effet prévus par les théoriciens. Par exemple si l'on considère $\psi(3095)$ et $\psi(3684)$ comme des états liés de quarks charmés, des états intermédiaires $0^{+}, 1^{+}, 2^{+}$devraient être créés.

Le $\psi(3684)$ a cependant une autre part de mystère. Que sont en effet les transitions qui ne sont pas des cascades? Parmi les $43 \%$ d'événements restants, quelques transitions du même type que celles du $\psi(3095)$ ont été identifiées. Il s'agit principalement de $\psi(3684) \rightarrow \omega \pi \pi$. Il semble cependant impossible que ce genre de désintégration ( $\psi$-like) puisse expliquer davantage que la moitié des désintégrations restantes. On se trouve donc devant un grand mystère. Il est probable que pour certaines raisons, plus d'une particule neutre participe à ces désintégrations. Pour mesurer qualitativement le problème, il suffit de regarder attentivement la figure 20.

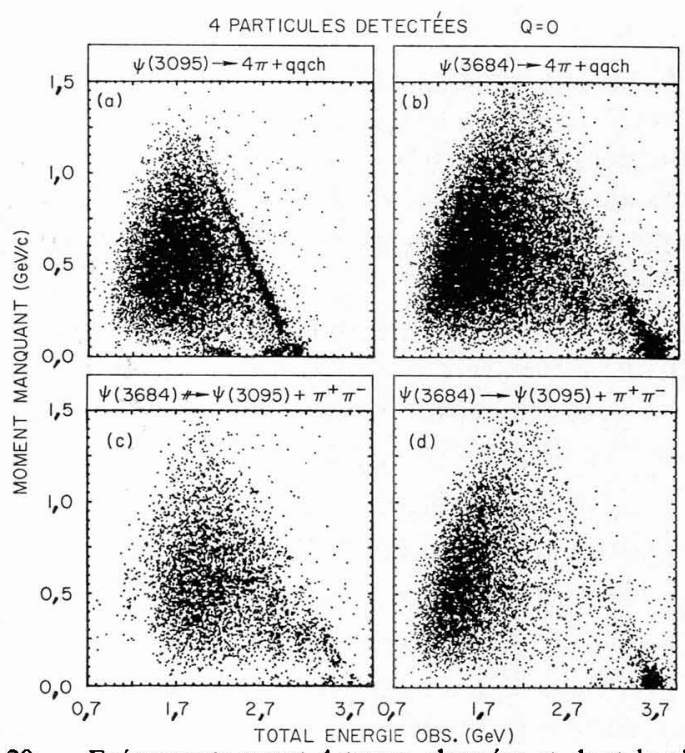

Fig. 20. - Evénements ayant 4 traces chargées et dont la charge totale est nulle : a) $\psi(3095) \rightarrow 4 \pi+\mathrm{X}$ énergie chargée détectée en fonction du moment manquant. b) $\psi(3684) \rightarrow 4 \pi+\mathrm{X}$ même distribution. c) $\psi(3684) \rightarrow \pi^{+} \pi^{-} \psi(3095)$ même distribution. d) $\psi(3684) \rightarrow 4 \pi+\mathrm{X}$ la distribution $c$ ) a été soustraite de $b$ ). On doit comparer les distributions $a$ ) et $d$ ). 
On s'intéresse à l'ensemble d'événements donnant 4 traces chargées dans le détecteur, et de charge totale nulle. La figure $20 a$ montre à $\psi(3095)$ la distribution de l'énergie chargée observée du système de 4 particules (on suppose que ce sont des pions) en fonction du moment manquant. 'Quand celui-ci est inférieur à $100 \mathrm{MeV} / \mathrm{c}$, on identifie les événements venant des modes $4 \pi, \pi \pi \mathrm{KK}, \pi \pi \mathrm{p} \overline{\mathrm{p}}$. Ces deux derniers apparaissent en effet à plus basse énergie car l'hypothèse de masse est fausse pour eux.

On aperçoit également la ligne dense des événements $4 \pi^{ \pm} \pi^{0}$. La figure $20 b$ montre les données du $\psi(3684)$ traitées de la même manière. Si l'on soustrait les événements $\psi(3684) \rightarrow \pi^{+} \pi^{-}+$quelque chose $(20 c)$, on obtient le spectre $20 d$. Celui-ci devrait se comparer (à la soustraction du mode $\psi\left(3684 \rightarrow \pi^{0} \pi^{0}\right.$ $\psi(3095)$ près $)$ à la figure $20 a$. Les différences sont flagrantes. Les événements $4 \pi, \pi \pi \mathrm{KK}, \pi \pi \mathrm{p} \overline{\mathrm{p}}$ sont certes présents mais en faible nombre.

De même les événements correspondant aux $4 \pi^{ \pm} \pi^{0}$ que l'on voit en $20 a$ sont à peine visibles, si l'on excepte ceux qui s'accumulent entre 100 et $300 \mathrm{MeV} / \mathrm{c}$.

Il est bien évident que si la part de mystère des désintégrations du $\psi(3684)$ correspond à des causes physiques nouvelles, elles restent encore à trouver.

6. Conclusion. - A l'heure actuelle, et comme il arrive souvent en physique, la découverte des $\psi$ et l'étude de la section efficace d'annihilation posent plus de problèmes qu'elles n'apportent de solutions. Il semble cependant qu'avec l'agitation fébrile qui s'est emparée de la physique des hautes énergies, on devrait assez rapidement trouver la solution des énigmes nouvelles.

\section{Bibliographie}

[1] SPEAR Storage Ring Group. Proceedings of the IXth International Conference on High Energy Accelerators, Stanford Linear Accelerator Center (1974) 37-42.

[2] Augustin, J. E. et al., Phys. Rev. Lett. 33 (1974) 1406.

[3] Aubert, J. J. et al., Phys. Rev. Lett. 33 (1974) 1404. Ces auteurs appellent la particule J (3 100).

[4] Abrams, G. S. et al., Phys. Rev. Lett. 33 (1974) 1433.

[5] Whitmore, J., Phys. Rep. 10C (1974) 273.

[6] PerL, M. L., Lectures on Electron Positron Annihilation. Part II : Anomalous Lepton Production. Institute of Particle Physics Summer School, McGill Univ. (1975).
[7] Boyarski, A. M. el al., Phys. Rev. Letl. 35 (1975) 196.

[8] Sokholov et Ternov, Sov. Phys. Dokl. 8-12 (1964) 1203.

[9] Augustin, J. E. et al., Phys. Rev. Lelt. 34 (1975) 233.

[10] Boyarski, A. M. et al., Phys. Rev. Lett. 34 (1975) 1357.

[11] Luith, V. et al., Phys. Rev. Lett. 35 (1975) 1124. LìTH, V., International Conference on High Energy Physics Palerme, 23-28 June 1975.

[12] Jfan-Marie. B. el al.. Phis. Rev. Lett. 36 (1976) 291.

[13] Aвrams, G. S. et al., Phys. Rev. Lett. 34 (1975) 1177. 